\title{
Protein kinase N2 connects blood flow with NO production in a double AKT
}

\author{
David J.R. Fulton and David W. Stepp \\ Vascular Biology Center at the Medical College of Georgia at Augusta University, Augusta, Georgia, USA.
}

\begin{abstract}
Shear stress is an important regulator of blood flow, and luminal endothelial cells (ECs) sense increases in frictional forces and respond with an appropriate release of vasoactive mediators. In this issue of the $J C I$, Jin et al. identified a mechanism by which ECs respond to shear stress with endothelial NOS (eNOS) activation and NO release. The authors showed that PKN2 was activated by fluid shear stress and contributed to eNOS activation via a double play - indirect phosphorylation at serine 1177 (S1177) via AKT and direct phosphorylation of the S1179 site. Phosphorylation of both sites individually increased eNOS activity, but together they had an additive effect. In sum, these findings reveal exciting details about how shear stress regulates eNOS and have important implications for blood flow and blood pressure.
\end{abstract}

activity. Moreover, a double D mutant of S1177D, S1179D, yielded an additive effect on NO production. Conversely, mutation of these sites to the phospho-null residue alanine had the opposite effect on eNOS activity. Using gene silencing, AKT1 was shown to mediate $\mathrm{p}-\mathrm{S} 1177$, but not $\mathrm{p}-\mathrm{S} 1179$, and PKN2 and its upstream kinase phosphoinositide-dependent protein kinase 1 (PDK1) were responsible for the double phosphorylation of S1177 and S1179. Activation ofPiezo1 and PDK1 were initiating events in the shear-induced regulation of p-PKN2, p-PDK1, and p-eNOS at S1177 and S1179. Shear-induced changes in p-PKN2 and p-PDK1 were dependent on changes in calcium, but not on phosphoinositide 3-kinase (PI3K), revealing a mechanism distinct from that previously described (4). AKT is activated by phosphorylation at two major regulatory sites, and $\mathrm{mTORC} 2$ was responsible for $\mathrm{p}-\mathrm{S} 473$ on $\mathrm{AKT}$, whereas PKN2 was responsible for increased p-T308 on AKT. Constitutively active PKN2 increased eNOS p-S1177 and p-S1179 and NO, independent of shear stress, and these responses were partly dependent on AKT p-T308. PKN2 physically interacted with eNOS and directly phosphorylated only S1179. In mice, EC deletion of PKN2 decreased both p-eNOS, impaired flowbut not acetylcholine-induced relaxation, and increased blood pressure. Collectively, this study provides a substantial body of in vitro and in vivo data demonstrating the ability of PKN2 to directly phosphorylate S1179 on eNOS and indirectly phosphorylate S1177 via AKT (Figure 1A). This double act fills in details in our understanding of how shear stress signals in ECs and how phosphorylation regulates eNOS.

\section{PKN2 mediates shear stress-} dependent signaling in ECs

Little is known about the functional role of PKN2 in the cardiovascular system (5). PKN2 belongs to a family of protein kinases (PKNs), formerly referred to as protein kinase C-related kinases, or PRKs. There 
A

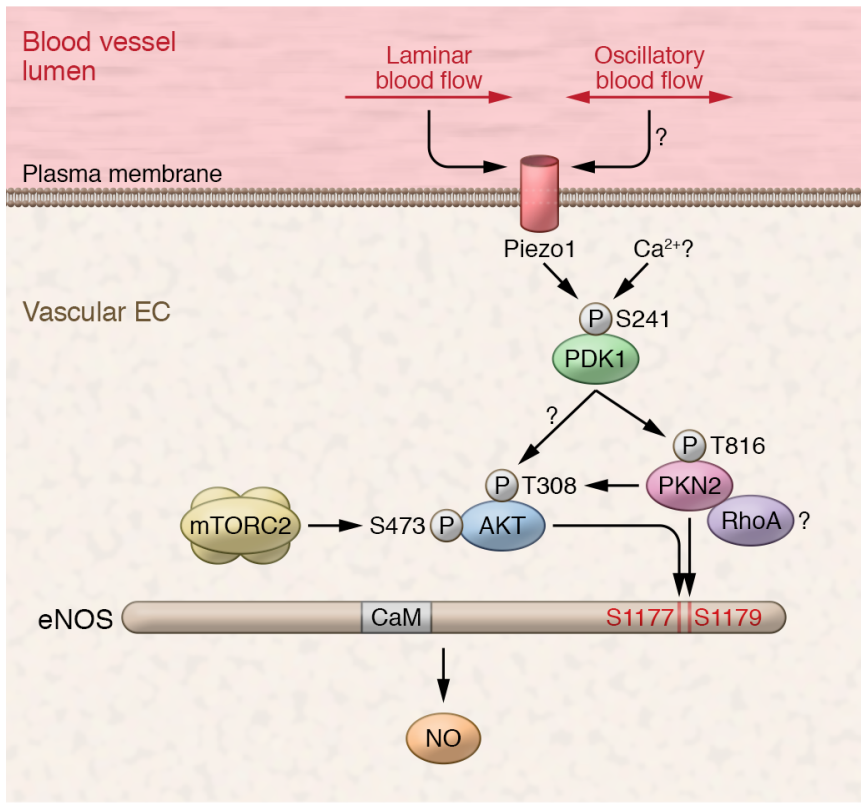

B

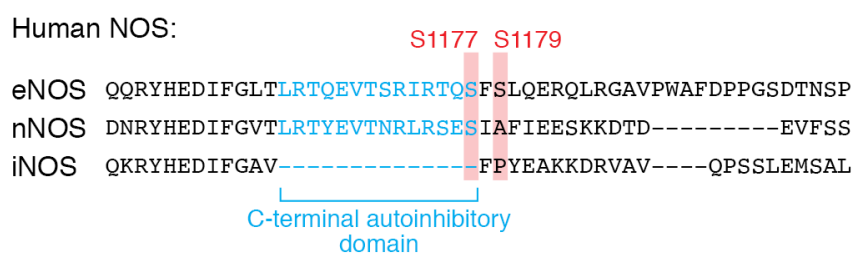

C

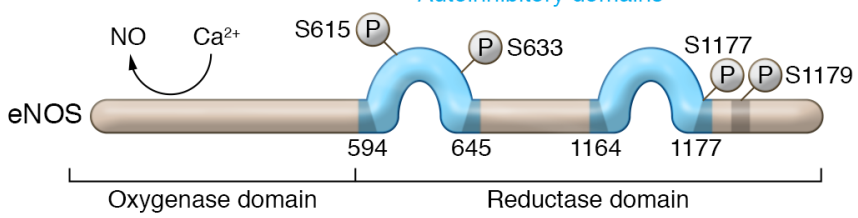

Figure 1. Mechanisms that regulate shear stress signaling and eNOS in ECs. (A) Acute laminar shear stress activates the mechanosensor Piezo1, triggering the release of ATP and the activation of P2Y which together promote the activating phosphorylation of PDK1 in a manner dependent on calcium $\left(\mathrm{Ca}^{2+}\right)$. How calcium contributes to the phosphorylation of PDK1 is not yet known. p-PDK1 then phosphorylates PNK2 at T816, resulting in the phosphorylation of AKT1 at T308, which leads to indirect phosphorylation of eNOS at S1177, an activating site (16), as well to direct phosphorylation of eNOS at S1179, a site described by Jin et al. (3) that increases eNOS activity. (B) Alignment of the C-termini of three human NOS (panel B was adapted from data in ref. 14). The calcium-dependent NOS isoforms (eNOS and nNOS) possess a C-terminal autoinhibitory domain that contains S1177 (eNOS) and an equivalent residue on nNOS, and both the autoinhibitory domain and internal phosphorylation site are absent in calcium-insensitive iNOS. S1179 is present in eNOS, but not nNOS or iNOS, suggesting a mechanism unique to eNOS. (C) S1179 lies just outside of the C-terminal autoinhibitory domain on human eNOS, which acts to facilitate calcium/CaM-dependent activation.

are three PKN isoforms $(1-3,6)$ that have $\mathrm{C}$-terminal serine/threonine kinase and calcium-binding $\mathrm{C} 2$ domains with homology to PKCs. The N-terminus of PKNs is distinguished by three polybasic coiled-coil homology region 1 (HR1) sites that bind to RhoA and Rho family members (5). Global KO of PKN2 is embryonically lethal, with severe consequences for vascular development (6). Surprisingly, mice with selective EC KO of PKN2 were viable, suggesting that PKN2 has critical developmental roles in other cell types. More subtle changes in endothelial and cardiovascular function with EC-specific PKN2 KO become observable in mature mice, as shown by Jin et al. (3). Shear stress promotes RhoA-dependent signaling in ECs, which contribute to structural changes in the endothelial cytoskeleton that align ECs with the direction of flow (7). While RhoA did not appear to contribute to the short-term activation of PKN2 in vitro in the study by Jin et al. (3), RhoA may have longer-term roles in EC cytoskeletal remodeling and polarization, as suggested by the in vivo data for the PKN2 EC-KO mice.

Jin et al. propose a signaling pathway by which the mechanosensor Piezo1 promotes the elevation of calcium, which increases the activating phosphorylation of PDK1. PDK1 binds PKN2 and phosphorylates T816 within its activation loop to increase PKN2 activity $(8,9)$. Other phosphorylation sites on PKN2 may also regulate its activity. For example, mTORC2 can phosphorylate PKN2 at T958, which has been proposed to increase PKN2 activity (10). Jin and colleagues showed that depletion of Rictor (mTORC2) did not affect PKN2 T816 phosphorylation (3), however, other sites may be involved. Future studies are needed to clarify how shear stress activates PKN2, with a focus on the kinase activity of PKN2 and accompanying changes in phosphorylation.

What drives the phosphorylation of PDK1 and PKN2? Jin et al. propose calcium as the driving mechanism, based on the reciprocal ability of BAPTA [1,2-Bis(2-aminophenoxy)ethane- $N, N, N^{\prime}, N^{\prime}$-tetraacetic acid tetrakis(acetoxymethyl ester)] and a calcium ionophore to regulate the phosphorylation of PDK1 at S241 and PKN2 at T816 (3). These data raise important questions about the identity of the calcium-regulated kinase(s) (or phosphatases) responsible for p-PDK1. There are also important differences to be resolved between shear stress- and agonist-mediated (acetylcholine-mediated) calcium mobilization, as only shear stress seems to activate PKN2/eNOS signaling (3).

\section{Direct and indirect PKN2 actions stimulate NO release}

Another important function of PKN2 is the activation of AKT. AKT is activated in ECs by shear stress, but the mechanisms involved have not been clearly defined. Prior studies reported that PKN1 and PKN2 inhibit AKT T308 phosphorylation (11), and fragments of PKN2 can alter the specificity of PDK1 so that it phosphory- 
lates AKT at S473 as well as at T308 (12). Jin and researchers used shear stress, an allosteric activator of PDK1, and a constitutively active form of PKN2 to show that PDK1 phosphorylated PKN2 at T816, and PKN2 phosphorylated T308 on AKT1 (3). Although these are compelling data, others have clearly shown that recombinant PDK1 can directly phosphorylate T308 on AKT (13), leaving a question as to why PKN2 is needed to achieve the same result. This interesting relationship requires further clarification. Another key finding was the ability of PKN2 to not only activate eNOS indirectly via AKT-dependent phosphorylation of S1177, but also to directly phosphorylate eNOS at S1179, a previously uncharacterized site. How S1179 phosphorylation regulates eNOS activity is not yet known. eNOS has a C-terminal NADPH binding reductase domain and an $\mathrm{N}$-terminal oxygenase domain that are separated by a canonical calcium-calmodulin ( $\mathrm{CaM})$ binding site. When activated by $\mathrm{CaM}$, the two domains align to allow electron flow and the synthesis of NO. Phosphorylation controls eNOS activity (primarily) by displacing mid- and C-terminal autoinhibitory loops to facilitate calcium/CaM-dependent activation. Deletion of these loops makes eNOS calcium independent, similar to the constitutively active inducible NOS (iNOS) (14). While a serine equivalent to $\mathrm{S} 1177$ is found in calcium-dependent neuronal NOS (nNOS), it is absent in calcium-independent iNOS (Figure 1B). S1179 lies just outside of the C-terminal autoinhibitory loop. There is no residue equivalent to $\mathrm{S} 1179$ in nNOS or iNOS, which presumably makes this mechanism of activation specific to eNOS. Given the close proximity, it is tempting to speculate that p-S1179 interacts with the C-ter- minal autoinhibitory loop (Figure 1C), but the additive effects of S1177D and S1179D on eNOS activity suggest that another mechanism awaits discovery.

\section{Future directions}

Jin et al. provide a major advance in our knowledge of shear stress-induced signaling in ECs and the regulation of AKT and eNOS activity and, in doing so, also generate exciting questions. How is PDK1 activated by shear stress and the role of calcium? What are the other substrates of PKN2 in ECs? Several studies have shown that PKN2 can alter cellular metabolism $(10,15)$, and whether this contributes to EC function remains to be investigated. The eNOS phosphorylation site at S1179 raises questions about whether phosphorylation of this site is altered by exercise or disease and whether there are other kinases that can phosphorylate this site. Future studies are needed to determine the roles of PKN2 in laminar versus turbulent shear (Figure 1A) and in the setting of atherosclerosis.

\section{Acknowledgments}

The authors are supported by grants from the NIH (1R01HL147159-0) and the American Heart Association (19TPA34910080).

Address correspondence to: David J.R. Fulton, Medical College of Georgia at Augusta University, 1460 Laney Walker Blvd, Augusta, Georgia 30907, USA. Email: dfulton@ augusta.edu.

1. Roux E, et al. Fluid shear stress sensing by the endothelial layer. Front Physiol. 2020;11:861.

2. Fulton DJR. Transcriptional and posttranslational regulation of eNOS in the endothelium. In: Khalil RA, ed. Advances in Pharmacology. Academic Press; 2016:29-64.

3. Jin YJ, et al. Protein kinase N2 mediates flow-induced endothelial NOS activation and vascular tone regulation. J Clin Invest. 2021;131(21):e145734.

4. Dimmeler S, et al. Activation of nitric oxide synthase in endothelial cells by Akt-dependent phosphorylation. Nature. 1999;399(6736):601-605.

5. Marrocco V, et al. PKC and PKN in heart disease. J Mol Cell Cardiol. 2019;128:212-226.

6. Quétier I, et al. Knockout of the PKN family of rho effector kinases reveals a non-redundant role for PKN2 in developmental mesoderm expansion. Cell Rep. 2016;14(3):440-448.

7. Wojciak-Stothard B, Ridley AJ. Shear stress-induced endothelial cell polarization is mediated by Rho and Rac but not Cdc42 or PI 3-kinases. JCell Biol. 2003;161(2):429-439.

8. Flynn P, et al. Rho GTPase control of protein kinase C-related protein kinase activation by 3-phosphoinositide-dependent protein kinase. J Biol Chem. 2000;275(15):11064-11070.

9. Dong LQ, et al. Phosphorylation of protein kinase $\mathrm{N}$ by phosphoinositide-dependent protein kinase-1 mediates insulin signals to the actin cytoskeleton. Proc Natl Acad Sci U S A. 2000;97(10):5089-5094.

10. Wallroth A, et al. Protein kinase N controls a lysosomal lipidswitch to facilitate nutrient signalling via mTORC1. Nat Cell Biol. 2019;21(9):1093-1101.

11. Wick MJ, et al. Mechanism of phosphorylation of protein kinase B/Akt by a constitutively active 3-phosphoinositide-dependent protein kinase-1. J Biol Chem. 2000;275(51):40400-40406.

12. Balendran A, et al. PDK1 acquires PDK2 activity in the presence of a synthetic peptide derived from the carboxyl terminus of PRK2. Curr Biol. 1999;9(8):393-404.

13. Chu N, et al. The structural determinants of $\mathrm{PH}$ domain-mediated regulation of Akt revealed by segmental labeling. Elife. 2020;9:e59151.

14. Chen PF, Wu KK. Structural elements contribute to the calcium/calmodulin dependence on enzyme activation in human endothelial nitric-oxide synthase. J Biol Chem. 2003;278(52):52392-52400.

15. Ruby MA, et al. Protein kinase N2 regulates AMP kinase signaling and insulin responsiveness of glucose metabolism in skeletal muscle. Am J Physiol Endocrinol Metab. 2017;313(4):E483-E491.

16. Fulton D, et al. Regulation of endotheliumderived nitric oxide production by the protein kinase Akt. Nature. 1999;399(6736):597-601. 